\title{
Mise en évidence, synthèse et caractérisation de nouveaux composés : les chlorures doubles d'ammonium et de calcium anhydres ou hydratés
}

\author{
R. Tenu, J. Berthet, M.A. Etoh et J.J. Counioux \\ Laboratoire Hydrazines et Procédés, UMR 5179, UCBL, CNRS, ISOCHEM/SNPE 22, \\ Avenue Gaston Berger, 69622 Villeurbanne Cedex, France
}

\begin{abstract}
The study of materials with phase change, often indicated PCM, has been developed because of their storage capacity of calorific or refrigerating energy. Their applications relate to the habitat, the transport, the protection of the electronic circuits exposed to strong variations in temperature or to the conservation of deep-frozen foods. A systematic study of the ternary system $\mathrm{H}_{2} \mathrm{O}-\mathrm{NH}_{4} \mathrm{Cl}-\mathrm{CaCl}_{2}$ was then undertaken at various temperatures by using different techniques like Isoplethic Thermal Analysis (I.T.A.), T.G.A., D.T.A. and X-Ray diffraction. Five invariant transformations and three new ternary compounds are observed within the temperature range $-20 / 70^{\circ} \mathrm{C}$. Finally, it appears that the calcium and ammonium chlorides form a rich family of anhydrous and hydrated double salts.
\end{abstract}

\section{INTRODUCTION}

L'étude des matériaux à changement de phase, souvent désignés MCP, s'est développée en raison de leur capacité de stockage de l'énergie calorifique ou frigorifique. Leurs applications concernent des secteurs aussi variés que l'habitat, le transport, la protection des circuits électroniques exposés à de fortes variations de température ou encore celle de la chaîne du froid des aliments surgelés. Dans tous les cas, les propriétés requises sont nombreuses et précises, ce qui rend difficile la mise au point de ces matériaux et par la suite leur extension.

Dans le domaine de l'habitat, un espoir avait été suscité par la possibilité d'utiliser le chlorure de calcium tétrahydraté pour stocker la chaleur et assurer des régulations thermiques. Ce sous-produit de l'industrie chimique, abondant et bon marché, présente en effet une décomposition péritectique à $45{ }^{\circ} \mathrm{C}$. D'une façon générale et bien que leur enthalpie de changement de phase puisse être importante, ces transformations non congruentes sont rarement retenues à des fins de stockage en raison de leur faible tenue en cyclage, inhérente aux phénomènes de ségrégations majeures dont ces matériaux sont l'objet. Il est alors nécessaire de recourir à un nouveau composé, présentant des caractéristiques économiques équivalentes, de façon à rechercher une combinaison à fusion congruente ou une eutexie quasi binaire ou ternaire. Le chlorure d'ammonium a ainsi été retenu.

Une étude bibliographique des équilibres entre phases du système ternaire $\mathrm{H}_{2} \mathrm{O}-\mathrm{NH}_{4} \mathrm{Cl}-\mathrm{CaCl}_{2}$ s'est révélée totalement infructueuse. Ce résultat est d'autant plus surprenant que les systèmes eau-chlorure alcalin-chlorure alcalino-terreux ont généralement été bien étudiés et depuis fort longtemps. Une étude systématique du système ternaire $\mathrm{H}_{2} \mathrm{O}-\mathrm{NH}_{4} \mathrm{Cl}-\mathrm{CaCl}_{2}$ a alors été entreprise au laboratoire à différentes températures. 


\section{RÉSULTATS EXPÉRIMENTAUX}

L'absence totale de données bibliographiques a rendu nécessaire l'étude d'une série d'isothermes qui ont été établies entre -20 et $70{ }^{\circ} \mathrm{C}$ par analyse thermique isopléthique (A.T.I.) [1]. La figure 1 représente à titre d'exemple les isothermes $-20,28$ et $50^{\circ} \mathrm{C}$.
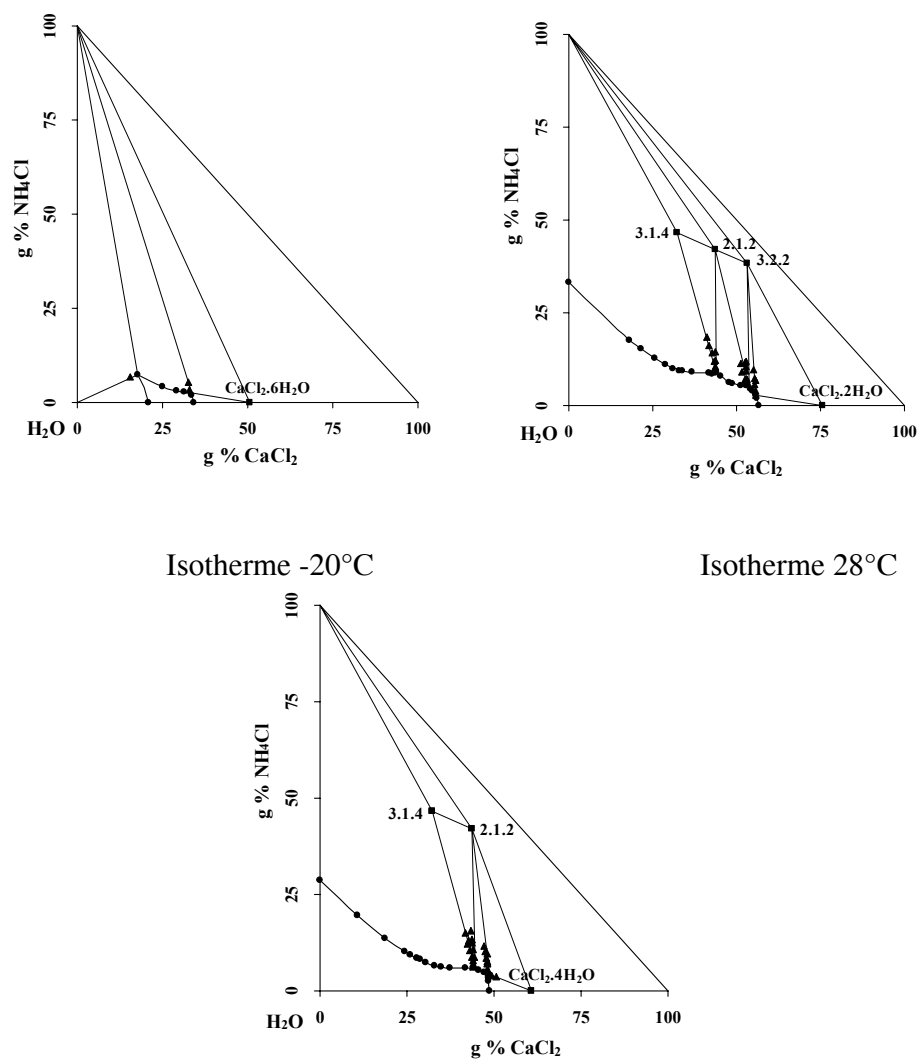

Isotherme $28^{\circ} \mathrm{C}$

Isotherme $50{ }^{\circ} \mathrm{C}$

Figure 1. Isothermes $-20,28$ et $50{ }^{\circ} \mathrm{C}$ du système ternaire $\mathrm{H}_{2} \mathrm{O}-\mathrm{NH}_{4} \mathrm{Cl}-\mathrm{CaCl}_{2}$.

Ces premiers travaux ont montré que :

- l'addition du chlorure d'ammonium ne permet pas de stabiliser le tétrahydrate du chlorure de calcium et par suite d'éviter les ségrégations majeures liées à la décomposition de cet hydrate.

- la complexité du système découle de l'existence de composés ternaires difficiles à observer et d'une zone de solution solide étendue.

- ce système, vraisemblablement étudié comme tous les ternaires du même type, n'avait jamais été publié certainement faute de résultats cohérents.

Afin de confirmer et approfondir les résultats obtenus par A.T.I., nous avons développé les recherches dans deux directions :

- l'étude du diagramme polythermique par analyse thermique

- l'étude des nouveaux composés par analyse thermogravimétrique et diffraction des rayons X. 
L'étude d'une série d'échantillons par analyse thermique à flux contrôlé a permis d'établir les caractéristiques des transformations invariantes du système ternaire jusqu'à $70{ }^{\circ} \mathrm{C}$. Une eutexie ternaire $\mathrm{E}$ et quatre transformations transitoires stables $\mathrm{T}_{1}, \mathrm{~T}_{2}, \mathrm{~T}_{3}$ et $\mathrm{T}_{4}$, se manifestent dans ce domaine de température. Leurs coordonnées sont indiquées dans le tableau 1.

Tableau 1. Coordonnées des solutions invariantes du système ternaire $\mathrm{H}_{2} \mathrm{O}-\mathrm{NH}_{4} \mathrm{Cl}-\mathrm{CaCl}_{2}$.

\begin{tabular}{|l|l|l|l|}
\hline \multirow{2}{*}{ Invariant } & \multicolumn{2}{|c|}{ Composition massique / g \% } & \multirow{2}{*}{$\mathrm{T} /{ }^{\circ} \mathrm{C}$} \\
\cline { 2 - 3 } & $\mathrm{CaCl}_{2}$ & $\mathrm{NH}_{4} \mathrm{Cl}$ & \\
\hline $\mathrm{E}$ & 30,4 & 0,8 & -50 \\
\hline $\mathrm{T}_{1}$ & 44,8 & 5,3 & 26 \\
\hline $\mathrm{T}_{2}$ & 49,5 & 3,9 & 28,5 \\
\hline $\mathrm{T}_{3}$ & 53,7 & 4,0 & 44 \\
\hline $\mathrm{T}_{4}$ & 55,7 & 2,5 & 45 \\
\hline
\end{tabular}

Les équilibres correspondants sont les suivants :
Eutexie E :
Liquide $\leftrightarrows$ Glace $+<$ S.S. $\mathrm{NH}_{4} \mathrm{Cl}>+<\mathrm{CaCl}_{2} \cdot 6 \mathrm{H}_{2} \mathrm{O}>$
Transformation transitoire $\mathrm{T}_{1}: \quad$ Liquide $+<2.1 .2>\leftrightarrows<\mathrm{S} . \mathrm{S} . \mathrm{NH}_{4} \mathrm{Cl}>+<\mathrm{CaCl}_{2} .4 \mathrm{H}_{2} \mathrm{O} \alpha>$
Transformation transitoire $\mathrm{T}_{2}: \quad$ Liquide $\left.+<\mathrm{CaCl}_{2} \cdot 4 \mathrm{H}_{2} \mathrm{O} \alpha\right\rangle \leftrightarrows<\mathrm{CaCl}_{2} \cdot 6 \mathrm{H}_{2} \mathrm{O}>+<2.1 .2>$
Transformation transitoire $\mathrm{T}_{3}: \quad$ Liquide $+<3.2 .2>\leftrightarrows<\mathrm{CaCl}_{2} \cdot 4 \mathrm{H}_{2} \mathrm{O} \alpha>+<2.1 .2>$
Transformation transitoire $\mathrm{T}_{4}: \quad$ Liquide $+<\mathrm{CaCl}_{2} \cdot 2 \mathrm{H}_{2} \mathrm{O}>\leftrightarrows<\mathrm{CaCl}_{2} \cdot 4 \mathrm{H}_{2} \mathrm{O} \alpha>+<3.2 .2>$

Les nouvelles phases solides mises en évidence par l'étude des équilibres solide-liquide du système ternaire sont une solution solide dérivée du chlorure d'ammonium notée $\mathrm{S} . \mathrm{S} \mathrm{NH}_{4} \mathrm{Cl}$ et trois chlorures doubles hydratés: $3 \mathrm{NH}_{4} \mathrm{Cl}_{2} \mathrm{CaCl}_{2} \cdot 4 \mathrm{H}_{2} \mathrm{O}, 2 \mathrm{NH}_{4} \mathrm{Cl} \cdot \mathrm{CaCl}_{2} \cdot 2 \mathrm{H}_{2} \mathrm{O}$ et $3 \mathrm{NH}_{4} \mathrm{Cl} \cdot 2 \mathrm{CaCl}_{2} \cdot 2 \mathrm{H}_{2} \mathrm{O}$ désignés respectivement par $<3.1 .4\rangle,<2.1 .2\rangle$ et $<3.2 .2>$.

Ces valeurs ont permis de tracer le diagramme polythermique représenté en projection sur le plan des compositions et en perspective cavalière dans la figure 2 .
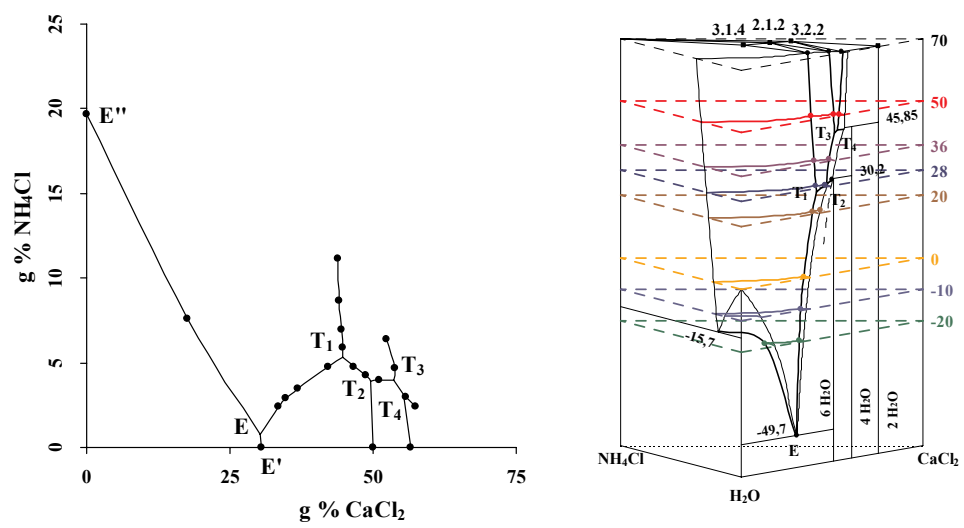

Figure 2. Diagramme polythermique du système ternaire $\mathrm{H}_{2} \mathrm{O}-\mathrm{NH}_{4} \mathrm{Cl}-\mathrm{CaCl}_{2}$ en projection sur le plan des compositions et en perspective cavalière.

La formation d'une solution solide par intégration d'ions $\mathrm{Ca}^{2+}$ et de molécules d'eau dans le réseau du chlorure d'ammonium II, cubique centré de groupe d'espace Pm3m [2], a été établie par analyse de l'évolution du paramètre de maille en fonction de la composition. Cette solution solide, valablement décrite par la formule $\left(\mathrm{NH}_{4} \mathrm{Cl}\right)_{1-\mathrm{x}}\left(\mathrm{CaCl}_{2} \cdot 4 \mathrm{H}_{2} \mathrm{O}\right)_{\mathrm{x}}$, traduit un caractère quasi-binaire partiel de la coupe 
isopléthique $\mathrm{NH}_{4} \mathrm{Cl}-\mathrm{CaCl}_{2} \cdot 4 \mathrm{H}_{2} \mathrm{O}$, le composé $<3.1 .4>$ pouvant alors être considéré comme une limite de stabilité de cette phase non stoechiométrique correspondant à $\mathrm{x}=1 / 4$.

En raison de la forte hygroscopicité des sels lorsqu'on les isole, l'étude approfondie des chlorures doubles hydratés a été limitée au composé $2 \mathrm{NH}_{4} \mathrm{Cl} \cdot \mathrm{CaCl}_{2} \cdot 2 \mathrm{H}_{2} \mathrm{O}$. Son élaboration a été assistée par A.T.I. en exploitant son domaine de cristallisation à $28^{\circ} \mathrm{C}$. Les cristaux ainsi obtenus sont fins, déliquescents et imprégnés d'eau-mère. La difficulté de les isoler se traduit par une composition chimique peu précise correspondant à la formule :

\section{$1,7 \mathrm{NH}_{4} \mathrm{Cl} \cdot \mathrm{CaCl}_{2} \cdot 2,8 \mathrm{H}_{2} \mathrm{O}$}

Pour les mêmes raisons, l'analyse par diffraction X de ces cristaux, protégés par un film de Kapton, montre toujours la présence de raies du chlorure d'ammonium sur le diffractogramme. Il a néanmoins été possible d'obtenir une série de raies significatives avec leurs positions et intensités relatives qui permettent d'identifier clairement ce composé (tableau 2).

L'analyse thermogravimétrique a confirmé la stoechiométrie du composé $<2.1 .2>$ et mis en évidence l'existence d'hydrates inférieurs et de chlorures doubles anhydres difficilement accessibles par d'autres voies de synthèse.

Après une phase de séchage des cristaux humides, le composé $<2.1 .2>$ se déshydrate par étapes, correspondant chacune au départ d'une demi molécule d'eau selon la séquence :

$$
\begin{array}{ll}
\text { - à } 110^{\circ} \mathrm{C}: & 2 \mathrm{NH}_{4} \mathrm{Cl} \cdot \mathrm{CaCl}_{2} \cdot 2 \mathrm{H}_{2} \mathrm{O} \rightarrow 2 \mathrm{NH}_{4} \mathrm{Cl} \cdot \mathrm{CaCl}_{2} \cdot 3 / 2 \mathrm{H}_{2} \mathrm{O} \\
\text { - à } 130^{\circ} \mathrm{C}: & 2 \mathrm{NH}_{4} \mathrm{Cl} \cdot \mathrm{CaCl}_{2} \cdot 3 / 2 \mathrm{H}_{2} \mathrm{O} \rightarrow 2 \mathrm{NH}_{4} \mathrm{Cl}^{\circ} \mathrm{CaCl}_{2} \cdot \mathrm{H}_{2} \mathrm{O} \\
\text { - à } 153^{\circ} \mathrm{C}: & 2 \mathrm{NH}_{4} \mathrm{Cl} \cdot \mathrm{CaCl}_{2} \cdot \mathrm{H}_{2} \mathrm{O} \rightarrow 2 \mathrm{NH}_{4} \mathrm{Cl}^{\circ} \mathrm{CaCl}_{2} \cdot 1 / 2 \mathrm{H}_{2} \mathrm{O} \\
\text { - à } 170^{\circ} \mathrm{C}: & 2 \mathrm{NH}_{4} \mathrm{Cl} \cdot \mathrm{CaCl}_{2} \cdot 1 / 2 \mathrm{H}_{2} \mathrm{O} \rightarrow 2 \mathrm{NH}_{4} \mathrm{Cl}^{\circ} \mathrm{CaCl}_{2}
\end{array}
$$

Vers $260{ }^{\circ} \mathrm{C}$, ce chlorure double anhydre se décompose à son tour pour former le composé $\mathrm{NH}_{4} \mathrm{Cl} . \mathrm{CaCl}_{2}$ par départ d'une molécule de chlorure d'ammonium.

\begin{tabular}{|c|c|c|c|c|c|}
\hline \multicolumn{2}{|c|}{$2 \mathrm{NH}_{4} \mathrm{Cl} \cdot \mathrm{CaCl}_{2} \cdot 2 \mathrm{H}_{2} \mathrm{O}$} & \multicolumn{2}{|c|}{$2 \mathrm{NH}_{4} \mathrm{Cl} . \mathrm{CaCl}_{2}$} & \multicolumn{2}{|c|}{$\mathrm{NH}_{4} \mathrm{Cl} \cdot \mathrm{CaCl}_{2}$} \\
\hline $2 \theta$ & $\mathbf{I} / \mathbf{I}_{0}$ & $2 \theta$ & $\mathbf{I} / \mathbf{I}_{0}$ & $2 \theta$ & $\mathbf{I} / \mathbf{I}_{\mathbf{0}}$ \\
\hline 15,80 & 15,1 & 22,53 & 16,7 & 15,58 & 13,7 \\
\hline 21,27 & 26,5 & 31,24 & 15,6 & 16,68 & 39,0 \\
\hline 31,58 & 100,0 & 32,02 & 100 & 16,88 & 86,4 \\
\hline 34,08 & 11,8 & 32,58 & 28,9 & 19,45 & 17,3 \\
\hline 39,41 & 23,0 & 39,46 & 10,2 & 21,11 & 18,2 \\
\hline 42,98 & 16,8 & 45,95 & 16,6 & 21,46 & 16,3 \\
\hline 47,18 & 16,0 & 57,10 & 15,6 & 23,79 & 17,1 \\
\hline 55,03 & 18,3 & & & 26,81 & 21,0 \\
\hline & & & & 27,88 & 23,6 \\
\hline & & & & 28,11 & 26,4 \\
\hline & & & & 33,66 & 81,0 \\
\hline & & & & 33,76 & 29,4 \\
\hline & & & & 34,01 & 100,0 \\
\hline & & & & 41,71 & 13,3 \\
\hline & & & & 41,97 & 16,0 \\
\hline & & & & 50,76 & 16,6 \\
\hline & & & & 51,56 & 11,1 \\
\hline
\end{tabular}

La formule des deux chlorures doubles anhydres $2 \mathrm{NH}_{4} \mathrm{Cl} . \mathrm{CaCl}_{2}(<2.1 .0>)$ et $\mathrm{NH}_{4} \mathrm{Cl} . \mathrm{CaCl}_{2}(<1.1 .0>)$ a été confirmée par analyse chimique et leurs diffractogrammes ont été établis (tableau 2).

Tableau 2. Raies les plus importantes obtenues par diffraction X. 


\section{CONCLUSION}

Finalement, il apparaît que les chlorures d'ammonium et de calcium forment une famille riche de sels doubles anhydres et hydratés, dont huit composés seulement sont mis en évidence à l'issue de ces travaux.

\section{Références}

[1] J. Berthet, J.J. Counioux,, Brevet F9313402

[2] Landolt Börnstein, volume 7a groupe III (1973) 248-350 